Kompass

Ophthalmologie

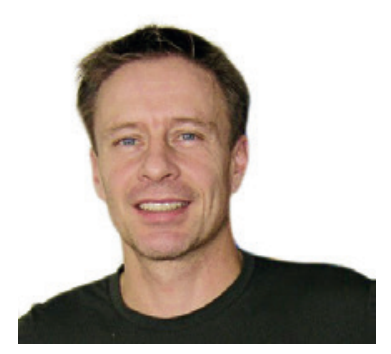

\title{
Fundus image analysis through a self-learning machine: Computer determines risk factors in hypertension
}

Olaf Strauß

Klinik für Augenheilkunde, Experimentelle Ophthalmologie, Charité - Universitätsmedizin Berlin, Campus Virchow-Klinikum, Berlin, Germany

Abstract of Poplin R, Varadarajan AV, Blumer K, et al.: Prediction of cardiovascular risk factors from retinal fundus photographs via deep learning. Nat Biomed Eng 2018;2:158-164.

\begin{abstract}
Traditionally, medical discoveries are made by observing associations, making hypotheses from them and then designing and running experiments to test the hypotheses. However, with medical images, observing and quantifying associations can often be difficult because of the wide variety of features, patterns, colours, values and shapes that are present in real data. Here, we show that deep learning can extract new knowledge from retinal fundus images. Using deep-learning models trained on data from 284,335 patients and validated on two independent datasets of 12,026
\end{abstract}

and 999 patients, we predicted cardiovascular risk factors not previously thought to be present or quantifiable in retinal images, such as age (mean absolute error within 3.26 years), gender (area under the receiver operating characteristic curve $(A \cup C)=0.97)$, smoking status ( $A \cup C=0.71$ ), systolic blood pressure (mean absolute error within $11.23 \mathrm{mmHg}$ ) and major adverse cardiac events ( $A \cup C=0.70$ ). We also show that the trained deep-learning models used anatomical features, such as the optic disc or blood vessels, to generate each prediction.

() 2019 Springer Nature Limited 


\section{Knowledge transfer}

\section{Background}

Artificial intelligence has arrived in ophthalmology. The annual conference of the German Ophthalmology Society is focusing on the topic in numerous scientific meetings. The objective is to improve diagnostics in many areas of image-aided analysis through the use of artificial intelligence.

\section{Study results}

The study by Poplin and colleagues from early 2018 in Nature Biomedical Engineering [1] represents a quantum leap here into the application of artificial intelligence in medicine in general and in ophthalmology in particular, which goes beyond a purely diagnostic application. Using about 300,000 fundus images of standardised quality taken from patients with hypertension, a self-learning algorithm "learned" to make correlations between special structural features of the fundus images and the cardiac risk factors of the patients, such as blood pressure, body mass index (BMI), age, or smoking. The homogeneous quality of the images as well as the patient documentation were important. Moreover, the machine was not given any specifications regarding what to search for - that is, it did not learn to look at the images as a physician would. In a second step, the machine assessed 13,000 fundus images. As a result, the machine was able to accurately determine the blood pressure, BMI, $\mathrm{HbA1c}$ or smoking status and, additionally, even reliably determine the gender of the patient from the images. In addition, the machine indicated which special structural features were used for these results. The interesting point in this study is that the authors had a group of experienced ophthalmologists repeat the same test on 100 fundus images. A table compares which structural features are the crucial factor for the ophthalmologists and which are the crucial factor for the machine. This shows, on the one hand, large similarities between human and machine, such as the blood vessels as a crucial feature. However, the machine took a number of other features into account, which were not considered by the ophthalmologists. The quantum leap is in the fact that there are features which cannot be learned through medical training but which are nonetheless dependent on the risk factors of hypertension. This means that the machine helps not only in detecting the features, but through the larger number of features considered, it also helps make a better diagnosis. The important question is why certain features, which previously received little or no consideration, react to the risk factors of hypertension. These may be indications for new pathomechanisms.

\section{Conclusions for clinical practice}

For the hospital, the use of self-learning machines yields not only the possibility of improving diagnoses. There is also the possibility of better understanding diseases, developing biomarkers, or the more accurate documentation of disease courses and thus, ultimately, improving therapy. Another paper from the group showed that refractive errors can be calculated from the fundus images [2]. In this way, the general diagnostic procedures could also be simplified, because much more information can be generated from fewer methodological steps.

\section{Disclosure Statement}

I hereby confirm that there are no conflicts of interest with regard to this commentary.

\section{Republication}

This article was first published in Karger Kompass Ophthalmol 2019; 5:166-167.

\section{References}

1 Poplin R, Varadarajan AV, Blumer K, et al.: Prediction of cardiovascular risk factors from retinal fundus photographs via deep learning. Nat Biomed Eng 2018:2:158-164.

2 Varadarajan AV, Poplin R, Blumer K, et al.: Deep learning for predicting refractive error from retinal fundus images. Invest Ophthalmol Vis Sci 2018;59: 2861-2868.

Kontaktadresse: Prof. Dr. Olaf Strauß, Klinik für Augenheilkunde, Experimentelle Ophthalmologie, Charité - Universitätsmedizin Berlin, Campus Virchow-Klinikum, Augustenburger Platz 1, 15333 Berlin, Germany, olaf. strauss@charite.de 